\title{
Transportation Energy Use: Comparison Including and Excluding Upstream Energy Use
}

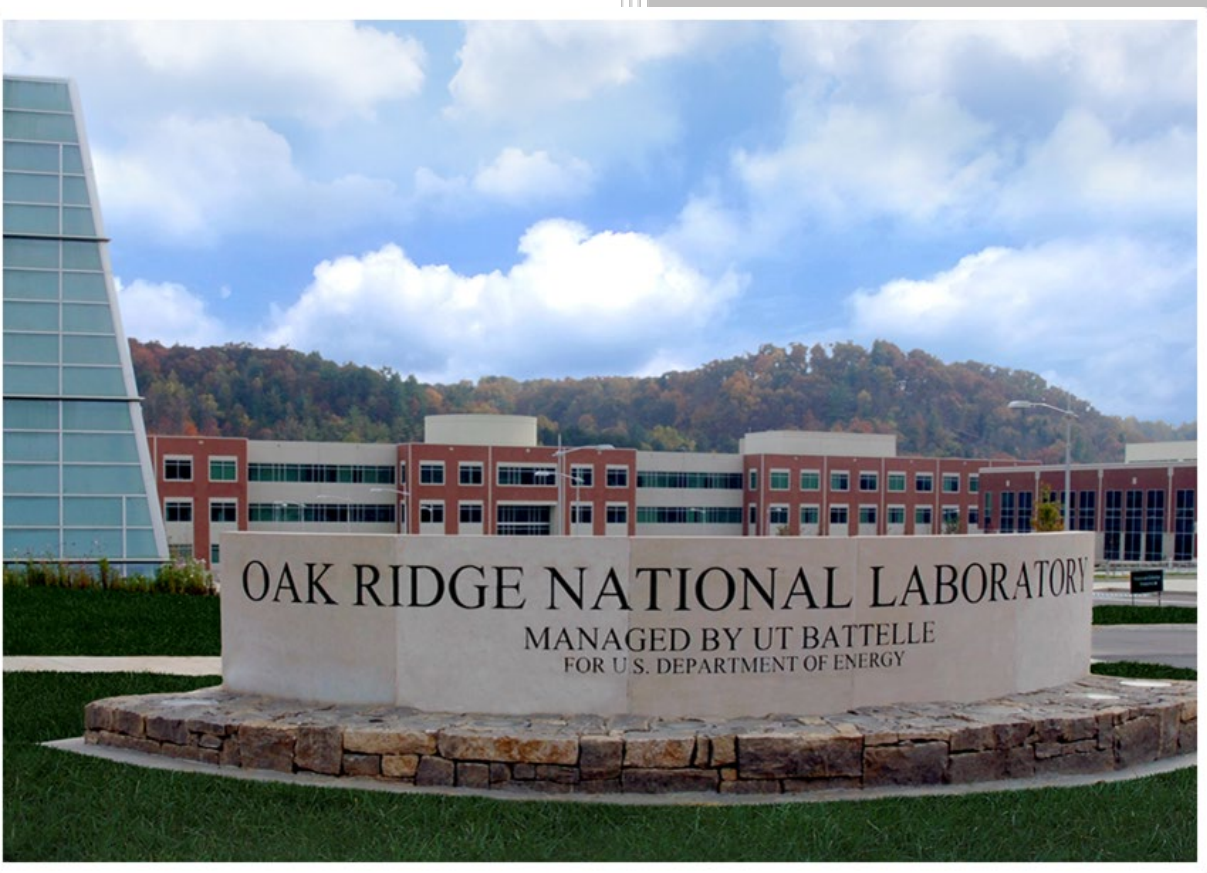

Stacy C. Davis

April 30, 2020 
This report was prepared as an account of work sponsored by an agency of the United States Government. Neither the United States Government nor any agency thereof, nor any of their employees, makes any warranty, express or implied, or assumes any legal liability or responsibility for the accuracy, completeness, or usefulness of any information, apparatus, product, or process disclosed, or represents that its use would not infringe privately owned rights. Reference herein to any specific commercial product, process, or service by trade name, trademark, manufacturer, or otherwise, does not necessarily constitute or imply its endorsement, recommendation, or favoring by the United States Government or any agency thereof. The views and opinions of authors expressed herein do not necessarily state or reflect those of the United States Government or any agency thereof. 
Energy and Transportation Science Division

TRANSPORTATION ENERGY:

\title{
A COMPARISON INCLUDING AND EXCLUDING UPSTREAM ENERGY
}

\author{
Stacy C. Davis
}

September 2020

Prepared by

OAK RIDGE NATIONAL LABORATORY

Oak Ridge, TN 37831-6283

managed by

UT-BATTELLE, LLC

for the

US DEPARTMENT OF ENERGY

under contract DE-AC05-00OR22725 



\section{CONTENTS}

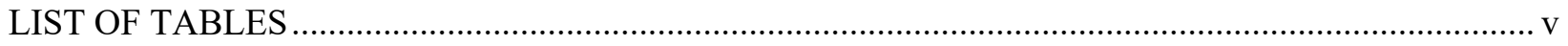

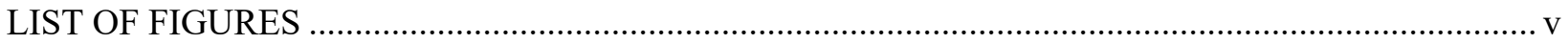

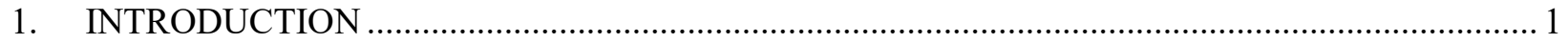

2. TRANSPORTATION VEHICLE ENERGY USE …..................................................................... 2

3. TRANSPORTATION ENERGY USE INCLUDING VEHICLE ENERGY USE PLUS

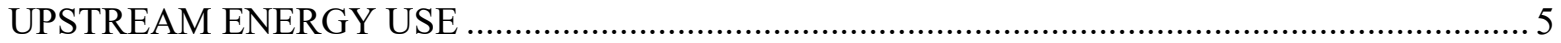

4. COMPARISON OF TRANSPORATION ENERGY CONSUMPTION WITH AND

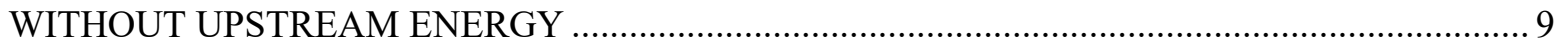

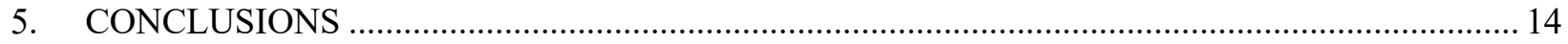




\section{LIST OF TABLES}

Table 1. Approximate Heat Rates and Heat Content of Electricity, 1970-2018 .................................... 1

Table 2. Domestic Consumption of Transportation Energy by Mode and Fuel Type, 2017 .................... 2

Table 3. Highway Transportation Energy Consumption by Mode, 1970-2017 …................................ 3

Table 4. Nonhighway Transportation Energy Consumption by Mode, 1970-2017 ................................ 4

Table 5. Factors for Including Upstream Energy Consumption ...................................................... 5

Table 6. Domestic Consumption of Transportation Energy Including Upstream Energy

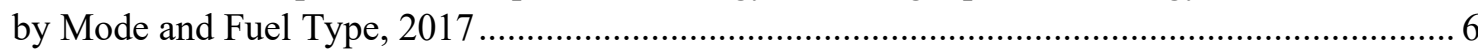

Table 7. Highway Transportation Energy Consumption Including Upstream Energy

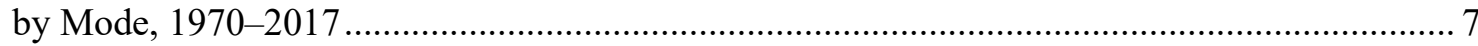

Table 8. Nonhighway Transportation Energy Consumption Including Upstream Energy

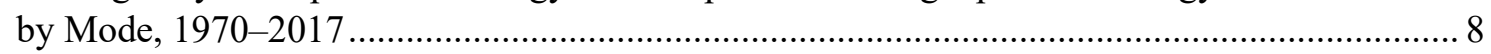

Table 9. Transportation Energy Use With and Without Upstream Energy by Mode, 2017 ................... 10

\section{LIST OF FIGURES}

Figure 1. Transportation energy consumption without upstream energy included................................. 9

Figure 2. Transportation energy consumption with upstream energy included..................................... 9

Figure 3. Percent change of vehicle energy use to energy use including upstream energy by mode, 2017.

Figure 4. Percent change of vehicle energy use to energy use including upstream energy by fuel type, 2017.

Figure 5. Historical transportation energy consumption with and without upstream energy, 1970-2017. 


\section{INTRODUCTION}

Thirty-eight editions of the Transportation Energy Data Book (Data Book) have been published since the report began in 1976. It was designed as a desk-top reference of statistics and information that characterize transportation activity. The most recent Data Book, edition 38, has 226 tables and 70 figures in the body of the report, with an additional 51 tables in appendices.

One of the most-often used tables in the Data Book is referred to by the authors as "the big energy table." It has transportation energy use in British thermal units (Btu) by mode and fuel type. The data are compiled from about 20 different sources and combined to create a detailed picture of transportation energy use. From the first edition to edition 35 of the Data Book, electricity use in the big energy table was converted from the original kilowatt-hours $(\mathrm{kWh})$ to Btu using the net generation heat rate for fossil fuels (Table 1). In those days, electricity was used mainly by transit rail. As electricity began to be used in the highway modes with consumer sales of electric vehicles, that process was revisited. Since energy use in the Data Book's big energy table for all other fuels did not include the upstream energy to create the fuel, it seemed inconsistent to include upstream energy for generation of electricity. Beginning with edition 36, electricity use for the big energy table was converted from kilowatt hours to Btu using the heat content of electricity (Table 1). Using heat content instead of net generation reduced electricity use data by about two-thirds.

Feedback in recent years on the change in process for electricity led to an investigation of the differences in transportation energy use when adding estimates of upstream energy use to all the fuels used in transportation. This report documents the result of that effort.

Table 1. Approximate Heat Rates and Heat Content of Electricity, 1970-2018 (Btu per kilowatt-hour)

\begin{tabular}{ccc|ccc}
\hline Year & $\begin{array}{c}\text { Electricity Net } \\
\text { Generation Heat Rate }\end{array}$ & $\begin{array}{c}\text { Heat Content of } \\
\text { Electricity }\end{array}$ & Year & $\begin{array}{c}\text { Electricity Net } \\
\text { Generation Heat Rate }\end{array}$ & $\begin{array}{c}\text { Heat Content of } \\
\text { Electricity }\end{array}$ \\
\hline 1970 & 10,494 & 3,412 & 1995 & 10,312 & 3,412 \\
1971 & 10,478 & 3,412 & 1996 & 10,340 & 3,412 \\
1972 & 10,379 & 3,412 & 1997 & 10,213 & 3,412 \\
1973 & 10,389 & 3,412 & 1998 & 10,197 & 3,412 \\
1974 & 10,442 & 3,412 & 1999 & 10,226 & 3,412 \\
1975 & 10,406 & 3,412 & 2000 & 10,201 & 3,412 \\
1976 & 10,373 & 3,412 & 2001 & 10,333 & 3,412 \\
1977 & 10,435 & 3,412 & 2002 & 10,173 & 3,412 \\
1978 & 10,361 & 3,412 & 2003 & 10,125 & 3,412 \\
1979 & 10,353 & 3,412 & 2004 & 10,016 & 3,412 \\
1980 & 10,388 & 3,412 & 2005 & 9,999 & 3,412 \\
1981 & 10,453 & 3,412 & 2006 & 9,919 & 3,412 \\
1982 & 10,454 & 3,412 & 2007 & 9,884 & 3,412 \\
1983 & 10,520 & 3,412 & 2008 & 9,854 & 3,412 \\
1984 & 10,440 & 3,412 & 2009 & 9,760 & 3,412 \\
1985 & 10,447 & 3,412 & 2010 & 9,756 & 3,412 \\
1986 & 10,446 & 3,412 & 2011 & 9,716 & 3,412 \\
1987 & 10,419 & 3,412 & 2012 & 9,516 & 3,412 \\
1988 & 10,324 & 3,412 & 2013 & 9,541 & 3,412 \\
1989 & 10,432 & 3,412 & 2014 & 9,510 & 3,412 \\
1990 & 10,402 & 3,412 & 2015 & 9,319 & 3,412 \\
1991 & 10,436 & 3,412 & 2016 & 9,232 & 3,412 \\
1992 & 10,342 & 3,412 & 2017 & 9,213 & 3,412 \\
1993 & 10,309 & 3,412 & 2018 & 9,213 & \\
1994 & 10,316 & 3,412 & & & \\
\hline
\end{tabular}

Source: U.S. Department of Energy, Energy Information Administration, Monthly Energy Review, Washington, DC, March 2019, Table A6.

\footnotetext{
${ }^{\mathrm{a}}$ For fossil fuels and noncombustible renewable energy. Does not include nuclear energy generation.
} 


\section{TRANSPORTATION VEHICLE ENERGY USE}

The Data Book's big energy table includes light vehicles, buses, and medium/heavy trucks in the highway mode, and air, water, pipeline, and rail in the nonhighway mode. Fuel types include gasoline, diesel fuel, liquefied petroleum gas, jet fuel, residual fuel oil, natural gas, and electricity. Table 2 is the Data Book's big energy table with energy used by the vehicles, not including upstream energy use for any fuel. For 2017, total energy use is 26.6 quadrillion Btu (quads).

Table 2. Domestic Consumption of Transportation Energy by Mode and Fuel Type, 2017

\begin{tabular}{|c|c|c|c|c|c|c|c|c|}
\hline & Gasoline & $\begin{array}{c}\text { Diesel } \\
\text { fuel }\end{array}$ & $\begin{array}{c}\text { Liquefied } \\
\text { petroleum } \\
\text { gas }\end{array}$ & Jet fuel & $\begin{array}{l}\text { Residual } \\
\text { fuel oil }\end{array}$ & $\begin{array}{c}\text { Natural } \\
\text { gas }\end{array}$ & Electricity & Total $^{\text {b }}$ \\
\hline HIGHWAY & $15,495.3$ & $6,266.5$ & 75.5 & - & - & 24.6 & 6.8 & $21,868.7$ \\
\hline Light vehicles & $14,853.3$ & 445.9 & 53.9 & - & - & - & 6.6 & $15,359.8$ \\
\hline Cars & $6,297.2$ & 36.2 & & & & & 5.8 & $6,339.3$ \\
\hline Light trucks ${ }^{\mathrm{c}}$ & $8,498.8$ & 409.6 & 53.9 & & & & 0.8 & $8,963.2$ \\
\hline Motorcycles & 57.3 & & & & & & & 57.3 \\
\hline Buses & 9.9 & 185.0 & 0.6 & - & - & 24.6 & 0.2 & 220.3 \\
\hline Transit & 1.6 & 64.6 & 0.6 & & & 24.6 & 0.2 & 91.6 \\
\hline Intercity & & 37.3 & & & & & & 37.3 \\
\hline School & 8.3 & 83.1 & & & & & & 91.4 \\
\hline Medium/heavy trucks & 632.0 & $5,635.6$ & 21.0 & - & - & - & - & $6,288.6$ \\
\hline Class 3-6 trucks & 581.4 & 789.0 & 20.8 & & & & & $1,391.2$ \\
\hline Class 7-8 trucks & 50.6 & $4,846.6$ & 0.2 & & & & & $4,897.4$ \\
\hline NONHIGHWAY & 214.8 & 818.5 & - & $2,208.2$ & 669.5 & 743.9 & 104.7 & $4,723.2$ \\
\hline$\overline{\text { Air }}$ & 22.6 & - & - & $2,208.2$ & - & - & - & $2,230.8$ \\
\hline General aviation & 22.6 & & & 209.1 & & & & 231.7 \\
\hline Domestic air carriers & & & & $1,564.3$ & & & & $1,564.3$ \\
\hline International air carriers ${ }^{\mathrm{d}}$ & & & & 434.8 & & & & 434.8 \\
\hline Water & 170.4 & 290.5 & - & - & 669.5 & - & - & $1,130.4$ \\
\hline Freight & & 250.7 & & & 669.5 & & & 920.2 \\
\hline Recreational & 170.4 & 39.9 & & & & & & 210.3 \\
\hline Pipeline & - & - & - & - & - & 743.9 & 81.1 & 825.0 \\
\hline Rail & - & 513.3 & - & - & - & - & 23.6 & 536.9 \\
\hline Freight (Class I) & & 490.5 & & & & & & 490.5 \\
\hline Passenger & & 22.8 & & & & & 23.6 & 46.4 \\
\hline Transit & & & & & & & 15.9 & 15.9 \\
\hline Commuter & & 14.5 & & & & & 6.1 & 20.5 \\
\hline Intercity & & 8.3 & & & & & 1.7 & 10.0 \\
\hline $\begin{array}{l}\text { TOTAL HWY \& } \\
\text { NONHWY }^{\mathrm{c}}\end{array}$ & $15,688.3$ & $7,070.4$ & 75.5 & $2,208.2$ & 669.5 & 768.4 & 111.6 & $26,591.9$ \\
\hline
\end{tabular}

Source: Oak Ridge National Laboratory, Transportation Energy Data Book: Edition 38, ORNL/TM-2019/1333, February 2020, Table 2.7.

a Civilian consumption only. Totals may not include all possible uses of fuels for transportation (e.g., snowmobiles).

$\mathrm{b}$ Totals may not sum due to rounding.

c Two-axle, four-tire trucks.

${ }^{\mathrm{d}}$ One half of fuel used by domestic carriers in international operation. 
Historical energy summaries are also provided in the Data Book. Table 3 shows highway modes for 1970-2017 using the same vehicle energy use data as the previous table, and Table 4 shows nonhighway modes.

Table 3. Highway Transportation Energy Consumption by Mode, 1970-2017

\begin{tabular}{|c|c|c|c|c|c|c|c|c|c|c|c|}
\hline Year & Cars & $\begin{array}{l}\text { Light } \\
\text { trucks }\end{array}$ & $\begin{array}{c}\text { Light } \\
\text { vehicles } \\
\text { subtotal }\end{array}$ & $\begin{array}{c}\text { Motor- } \\
\text { cycles }\end{array}$ & Buses & $\begin{array}{c}\text { Class } \\
3-6 \\
\text { trucks }\end{array}$ & $\begin{array}{c}\text { Class } \\
7-8 \\
\text { trucks }\end{array}$ & $\begin{array}{c}\text { Heavy } \\
\text { trucks } \\
\text { subtotal }\end{array}$ & & $\begin{array}{c}\text { Highway } \\
\text { subtotal }\end{array}$ & $\begin{array}{c}\text { Total } \\
\text { transportation }^{\mathrm{a}}\end{array}$ \\
\hline 1970 & 8,479 & 1,539 & 10,018 & 7 & 129 & 333 & 1,220 & 1,553 & & 11,707 & 15,192 \\
\hline 1975 & 9,298 & 2,384 & 11,682 & 14 & 124 & 430 & 1,574 & 2,003 & & 13,823 & 17,204 \\
\hline 1976 & 9,826 & 2,602 & 12,428 & 15 & 134 & 453 & 1,661 & 2,114 & & 14,691 & 18,266 \\
\hline 1977 & 9,928 & 2,797 & 12,725 & 16 & 137 & 503 & 1,841 & 2,344 & & 15,222 & 18,951 \\
\hline 1978 & 10,134 & 3,020 & 13,154 & 18 & 141 & 672 & 1,935 & 2,607 & & 15,920 & 19,922 \\
\hline 1979 & 9,629 & 3,056 & 12,685 & 22 & 144 & 813 & 1,884 & 2,697 & & 15,548 & 19,473 \\
\hline 1980 & 8,800 & 2,975 & 11,775 & 26 & 143 & 929 & 1,757 & 2,686 & & 14,630 & 18,760 \\
\hline 1981 & 8,693 & 2,963 & 11,656 & 27 & 145 & 1,065 & 1,659 & 2,724 & & 14,552 & 18,558 \\
\hline 1982 & 8,673 & 2,837 & 11,510 & 25 & 151 & 1,182 & 1,525 & 2,707 & & 14,393 & 18,055 \\
\hline 1983 & 8,802 & 2,990 & 11,792 & 22 & 152 & 1,121 & 1,649 & 2,770 & & 14,736 & 18,188 \\
\hline 1984 & 8,837 & 3,197 & 12,034 & 22 & 146 & 1,072 & 1,801 & 2,873 & & 15,075 & 18,773 \\
\hline 1985 & 8,932 & 3,413 & 12,345 & 23 & 153 & 986 & 1,897 & 2,883 & & 15,404 & 19,017 \\
\hline 1986 & 9,138 & 3,629 & 12,767 & 23 & 160 & 920 & 2,038 & 2,958 & & 15,908 & 20,086 \\
\hline 1987 & 9,157 & 3,819 & 12,976 & 24 & 164 & 858 & 2,203 & 3,061 & & 16,225 & 20,578 \\
\hline 1988 & 9,158 & 4,078 & 13,236 & 25 & 169 & 860 & 2,257 & 3,118 & & 16,548 & 21,131 \\
\hline 1989 & 9,232 & 4,156 & 13,388 & 26 & 169 & 869 & 2,330 & 3,199 & & 16,782 & 21,487 \\
\hline 1990 & 8,688 & 4,451 & 13,139 & 24 & 167 & 891 & 2,442 & 3,334 & & 16,664 & 21,383 \\
\hline 1991 & 8,029 & 4,774 & 12,803 & 23 & 177 & 895 & 2,507 & 3,402 & & 16,405 & 20,985 \\
\hline 1992 & 8,169 & 5,117 & 13,286 & 24 & 184 & 897 & 2,570 & 3,468 & & 16,962 & 21,646 \\
\hline 1993 & 8,368 & 5,356 & 13,724 & 25 & 183 & 906 & 2,671 & 3,577 & & 17,509 & 22,125 \\
\hline 1994 & 8,470 & 5,515 & 13,985 & 26 & 183 & 936 & 2,842 & 3,778 & & 17,972 & 22,729 \\
\hline 1995 & 8,489 & 5,695 & 14,184 & 25 & 184 & 954 & 2,983 & 3,937 & & 18,330 & 23,263 \\
\hline 1996 & 8,634 & 5,917 & 14,551 & 24 & 186 & 958 & 3,088 & 4,045 & & 18,806 & 23,773 \\
\hline 1997 & 8,710 & 6,169 & 14,879 & 25 & 192 & 945 & 3,141 & 4,086 & & 19,182 & 24,126 \\
\hline 1998 & 8,936 & 6,303 & 15,239 & 26 & 196 & 967 & 3,251 & 4,218 & & 19,679 & 24,461 \\
\hline 1999 & 9,134 & 6,602 & 15,736 & 26 & 203 & 1,054 & 3,584 & 4,638 & & 20,603 & 25,760 \\
\hline 2000 & 9,100 & 6,607 & 15,707 & 26 & 209 & 1,085 & 3,734 & 4,819 & & 20,761 & 26,071 \\
\hline 2001 & 9,161 & 6,678 & 15,839 & 24 & 196 & 1,074 & 3,738 & 4,813 & & 20,872 & 25,741 \\
\hline 2002 & 9,391 & 6,883 & 16,274 & 24 & 192 & 1,114 & 3,921 & 5,035 & & 21,525 & 26,329 \\
\hline 2003 & 9,255 & 7,551 & 16,806 & 24 & 190 & 1,083 & 3,812 & 4,895 & & 21,915 & 26,509 \\
\hline 2004 & 9,331 & 7,861 & 17,192 & 25 & 194 & 1,003 & 3,532 & 4,535 & & 21,946 & 26,965 \\
\hline 2005 & 9,579 & 7,296 & 16,875 & 24 & 196 & 1,126 & 3,963 & 5,088 & & 22,183 & 27,373 \\
\hline 2006 & 9,316 & 7,550 & 16,866 & 28 & 199 & 1,149 & 4,045 & 5,193 & $b$ & 22,286 & 27,546 \\
\hline 2007 & 9,221 & 7,679 & 16,900 & 59 & 195 & 1,429 & 5,031 & 6,460 & & 23,615 & 29,004 \\
\hline 2008 & 8,831 & 7,572 & 16,404 & 61 & 200 & 1,444 & 5,083 & 6,527 & & 23,192 & 28,365 \\
\hline 2009 & 8,209 & 7,635 & 15,843 & 60 & 200 & 1,341 & 4,720 & 6,061 & & 22,165 & 26,878 \\
\hline 2010 & 7,657 & 7,971 & 15,628 & 53 & 190 & 1,363 & 4,797 & 6,160 & & 22,032 & 26,949 \\
\hline 2011 & 7,336 & 8,104 & 15,440 & 53 & 195 & 1,283 & 4,517 & 5,801 & & 21,489 & 26,357 \\
\hline 2012 & 7,121 & 8,180 & 15,300 & 61 & 200 & 1,282 & 4,512 & 5,794 & & 21,356 & 25,966 \\
\hline 2013 & 7,047 & 8,077 & 15,124 & 58 & 204 & 1,310 & 4,613 & 5,924 & & 21,310 & 25,868 \\
\hline 2014 & 6,951 & 8,506 & 15,454 & 57 & 206 & 1,332 & 4,689 & 6,022 & & 21,742 & 25,949 \\
\hline 2015 & 6,716 & 8,654 & 15,370 & 56 & 210 & 1,324 & 4,660 & 5,984 & & 21,619 & 26,084 \\
\hline 2016 & 6,577 & 8,890 & 15,467 & 58 & 214 & 1,359 & 4,783 & 6,142 & & 21,881 & 26,485 \\
\hline \multirow[t]{2}{*}{2017} & 6,339 & 8,963 & 15,302 & 57 & 220 & 1,391 & 4,897 & 6,289 & & 21,869 & 26,592 \\
\hline & & \multicolumn{10}{|c|}{ Average annual percentage change } \\
\hline $970-2017$ & $-0.6 \%$ & $3.8 \%$ & $0.9 \%$ & $4.6 \%$ & $1.1 \%$ & $3.1 \%$ & $3.0 \%$ & $3.0 \%$ & & $1.3 \%$ & $1.2 \%$ \\
\hline $007-2017$ & $-3.7 \%$ & $1.6 \%$ & $-1.0 \%$ & $-0.4 \%$ & $1.3 \%$ & $-0.3 \%$ & $-0.3 \%$ & $-0.3 \%$ & & $-0.8 \%$ & $-0.9 \%$ \\
\hline
\end{tabular}

Source: Oak Ridge National Laboratory, Transportation Energy Data Book: Edition 38, ORNL/TM-2019/1333, February 2020, Table 2.9.

a Total transportation figures do not include military and off-highway energy use and may not include all possible uses of fuel for transportation (e.g., snowmobiles).

${ }^{b}$ Due to changes in the FHWA fuel use methodology, motorcycle, bus, and heavy truck data are not comparable with data before the year 2007. Car and light truck data changed after 2008. 
Table 4. Nonhighway Transportation Energy Consumption by Mode, 1970-2017 (trillion Btu)

\begin{tabular}{|c|c|c|c|c|c|c|}
\hline Year & Air & Water & Pipeline & Rail & $\begin{array}{c}\text { Nonhighway } \\
\text { subtotal }\end{array}$ & $\begin{array}{c}\text { Total } \\
\text { transportation }^{\mathrm{a}} \\
\end{array}$ \\
\hline 1970 & 1,287 & 836 & 826 & 537 & 3,486 & 15,192 \\
\hline 1975 & 1,234 & 927 & 680 & 540 & 3,381 & 17,204 \\
\hline 1976 & 1,285 & 1,083 & 644 & 562 & 3,574 & 18,266 \\
\hline 1977 & 1,350 & 1,177 & 627 & 574 & 3,728 & 18,951 \\
\hline 1978 & 1,423 & 1,382 & 625 & 572 & 4,002 & 19,922 \\
\hline 1979 & 1,488 & 1,149 & 699 & 588 & 3,925 & 19,473 \\
\hline 1980 & 1,434 & 1,393 & 734 & 570 & 4,130 & 18,760 \\
\hline 1981 & 1,453 & 1,270 & 742 & 541 & 4,006 & 18,558 \\
\hline 1982 & 1,445 & 1,063 & 694 & 460 & 3,662 & 18,055 \\
\hline 1983 & 1,440 & 974 & 583 & 455 & 3,452 & 18,188 \\
\hline 1984 & 1,609 & 964 & 623 & 502 & 3,699 & 18,773 \\
\hline 1985 & 1,677 & 871 & 597 & 468 & 3,613 & 19,017 \\
\hline 1986 & 1,823 & 1,323 & 578 & 454 & 4,178 & 20,086 \\
\hline 1987 & 1,899 & 1,378 & 613 & 464 & 4,354 & 20,578 \\
\hline 1988 & 1,978 & 1,417 & 712 & 476 & 4,583 & 21,131 \\
\hline 1989 & 1,981 & 1,516 & 729 & 478 & 4,705 & 21,487 \\
\hline 1990 & 2,046 & 1,442 & 760 & 471 & 4,719 & 21,383 \\
\hline 1991 & 1,916 & 1,523 & 699 & 442 & 4,580 & 20,985 \\
\hline 1992 & 1,945 & 1,599 & 685 & 455 & 4,684 & 21,646 \\
\hline 1993 & 1,986 & 1,437 & 723 & 469 & 4,615 & 22,125 \\
\hline 1994 & 2,075 & 1,394 & 787 & 502 & 4,758 & 22,729 \\
\hline 1995 & 2,141 & 1,468 & 803 & 523 & 4,935 & 23,263 \\
\hline 1996 & 2,206 & 1,411 & 814 & 536 & 4,967 & 23,773 \\
\hline 1997 & 2,300 & 1,250 & 856 & 537 & 4,943 & 24,126 \\
\hline 1998 & 2,275 & 1,232 & 735 & 540 & 4,782 & 24,461 \\
\hline 1999 & 2,483 & 1,370 & 745 & 560 & 5,156 & 25,760 \\
\hline 2000 & 2,554 & 1,455 & 742 & 559 & 5,309 & 26,071 \\
\hline 2001 & 2,397 & 1,187 & 724 & 561 & 4,869 & 25,741 \\
\hline 2002 & 2,229 & 1,246 & 768 & 563 & 4,807 & 26,329 \\
\hline 2003 & 2,260 & 1,071 & 689 & 575 & 4,597 & 26,509 \\
\hline 2004 & 2,456 & 1,293 & 662 & 607 & 5,024 & 26,965 \\
\hline 2005 & 2,532 & 1,363 & 681 & 613 & 5,194 & 27,373 \\
\hline 2006 & 2,511 & 1,442 & 681 & 626 & 5,269 & 27,546 \\
\hline 2007 & 2,509 & 1,550 & 720 & 610 & 5,399 & 29,004 \\
\hline 2008 & 2,396 & 1,444 & 748 & 586 & 5,190 & 28,365 \\
\hline 2009 & 2,127 & 1,323 & 771 & 492 & 4,731 & 26,878 \\
\hline 2010 & 2,149 & 1,460 & 775 & 533 & 4,942 & 26,949 \\
\hline 2011 & 2,157 & 1,362 & 790 & 560 & 4,900 & 26,357 \\
\hline 2012 & 2,077 & 1,148 & 835 & 549 & 4,644 & 25,966 \\
\hline 2013 & 2,037 & 1,017 & 942 & 562 & 4,596 & 25,868 \\
\hline 2014 & 2,060 & 876 & 803 & 587 & 4,367 & 25,949 \\
\hline 2015 & 2,118 & 1,005 & 780 & 563 & 4,505 & 26,084 \\
\hline 2016 & 2,178 & 1,116 & 789 & 520 & 4,642 & 26,485 \\
\hline 2017 & 2,231 & 1,130 & 825 & 537 & 4,760 & 26,592 \\
\hline \multicolumn{7}{|c|}{ Average annual percentage change } \\
\hline $1970-2017$ & $1.2 \%$ & $0.7 \%$ & $0.0 \%$ & $0.0 \%$ & $0.7 \%$ & $1.2 \%$ \\
\hline $2007-2017$ & $-1.2 \%$ & $-2.9 \%$ & $1.4 \%$ & $-1.3 \%$ & $-1.3 \%$ & $-0.9 \%$ \\
\hline
\end{tabular}

Source: Oak Ridge National Laboratory, Transportation Energy Data Book: Edition 38, ORNL/TM-2019/1333, February 2020, Table 2.10.

a Total transportation figures do not include military and off-highway energy use and may not include all possible uses of fuel for transportation (e.g., snowmobiles). 


\section{TRANSPORTATION ENERGY USE INCLUDING VEHICLE ENERGY USE PLUS UPSTREAM ENERGY USE}

Argonne National Laboratory's Greenhouse gases, Regulated Emissions, and Energy use in Transportation (GREET) Model has a Well-to-Wheels (WTW) calculator which provides factors for the energy used to produce transportation fuels (upstream energy). Table 5 shows the factors by fuel type that were used to convert vehicle energy consumption to include upstream energy consumption for all fuels except electricity. Electricity consumption was converted to include upstream energy using the net generation heat rate on Table 1. The next three tables are the same as in section 2, but the data have upstream energy use included (Tables 6-8). Total transportation energy use including upstream energy was 33.0 quads in 2017.

Table 5. Factors for Including Upstream Energy Consumption

\begin{tabular}{lc}
\hline & Upstream Energy \\
Fuel Type & Factor \\
\hline Gasoline (E0) & 1.24 \\
Gasoline (E10) & 1.28 \\
Diesel & 1.19 \\
Biodiesel & 1.50 \\
CNG & 1.15 \\
LNG & 1.20 \\
LPG & 1.15 \\
Jet Fuel (conventional) & 1.15 \\
Marine Heavy Fuel Oil (2.7\% Sulfur) & 1.14 \\
\hline
\end{tabular}

Source: Argonne National Laboratory, GREET 2019 WTW Calculator, accessed March 6, 2020. 
Table 6. Domestic Consumption of Transportation Energy Including Upstream Energy by Mode and Fuel Type, 2017

\begin{tabular}{|c|c|c|c|c|c|c|c|c|}
\hline & Gasoline & $\begin{array}{l}\text { Diesel } \\
\text { fuel }\end{array}$ & $\begin{array}{l}\text { Liquefied } \\
\text { petroleum } \\
\text { gas }\end{array}$ & Jet fuel & $\begin{array}{l}\text { Residual } \\
\text { fuel oil }\end{array}$ & $\begin{array}{l}\text { Natural } \\
\text { gas }\end{array}$ & Electricity & Total $^{\mathrm{b}}$ \\
\hline HIGHWAY & $19,793.7$ & $7,458.6$ & 86.8 & - & - & 28.3 & 18.5 & $27,385.9$ \\
\hline$\overline{\text { Light vehicles }}$ & $18,997.7$ & 530.6 & 62.0 & - & - & - & 17.9 & $19,608.2$ \\
\hline Cars & $8,048.7$ & 43.1 & & & & & 15.6 & $8,107.4$ \\
\hline Light trucks ${ }^{\mathrm{c}}$ & $10,877.7$ & 487.5 & 62.0 & & & & 2.3 & $11,429.5$ \\
\hline Motorcycles & 71.3 & & & & & & & 71.3 \\
\hline Buses & 12.3 & 221.6 & 0.7 & - & - & 28.3 & 0.6 & 263.5 \\
\hline Transit & 2.0 & 78.3 & 0.7 & & & 28.3 & 0.6 & 109.9 \\
\hline Intercity & & 44.4 & & & & & & 44.4 \\
\hline School & 10.3 & 98.9 & & & & & & 109.2 \\
\hline Medium/heavy trucks & 783.7 & $6,706.4$ & 24.1 & - & - & - & - & $7,514.2$ \\
\hline Class 3-6 trucks & 721 & 938.9 & 23.9 & & & & & $1,683.8$ \\
\hline Class $7-8$ trucks & 62.7 & $5,767.50$ & 0.2 & & & & & $5,830.4$ \\
\hline NONHIGHWAY & 240.0 & 956.5 & - & $2,539.5$ & 763.2 & 890.1 & 274.2 & $5,663.5$ \\
\hline Air & 28.7 & - & - & $2,539.5$ & - & - & - & $2,568.2$ \\
\hline General aviation & 28.7 & & & 240.4 & & & & 269.1 \\
\hline Domestic air carriers & & & & $1,799.00$ & & & & $1,799.0$ \\
\hline International air carriers ${ }^{\mathrm{d}}$ & & & & 500.1 & & & & 500.1 \\
\hline Water & 211.3 & 345.7 & - & - & 763.2 & - & - & $1,320.2$ \\
\hline Freight & & 298.3 & & & 763.2 & & & $1,061.5$ \\
\hline Recreational & 211.3 & 47.4 & & & & & & 258.7 \\
\hline Pipeline & - & - & - & - & - & 890.1 & 219 & $1,109.1$ \\
\hline Rail & - & 610.8 & - & - & - & - & 55.2 & 666.0 \\
\hline Freight (Class I) & & 583.7 & & & & & & 583.7 \\
\hline Passenger & & 27.1 & & & & & 55.2 & 82.3 \\
\hline Transit & & & & & & & 34.3 & 34.3 \\
\hline Commuter & & 17.2 & & & & & 16.4 & 33.6 \\
\hline Intercity & & 9.9 & & & & & 4.5 & 14.4 \\
\hline $\begin{array}{l}\text { TOTAL HWY \& } \\
\text { NONHWY }^{\mathrm{c}}\end{array}$ & $20,033.7$ & $8,415.1$ & 86.8 & $2,539.5$ & 763.2 & 918.4 & 292.7 & $33,049.4$ \\
\hline
\end{tabular}

Source: Vehicle energy use from the Data Book plus upstream energy use for all fuels.

a Civilian consumption only. Totals may not include all possible uses of fuels for transportation (e.g., snowmobiles).

$\mathrm{b}$ Totals may not sum due to rounding.

c Two-axle, four-tire trucks.

${ }^{\mathrm{d}}$ One half of fuel used by domestic carriers in international operation. 
Table 7. Highway Transportation Energy Consumption Including Upstream Energy by Mode, 1970-2017

(trillion Btu)

\begin{tabular}{|c|c|c|c|c|c|c|c|c|c|c|c|}
\hline Year & Cars & $\begin{array}{l}\text { Light } \\
\text { trucks }\end{array}$ & $\begin{array}{c}\text { Light } \\
\text { vehicles } \\
\text { subtotal }\end{array}$ & $\begin{array}{c}\text { Motor- } \\
\text { cycles }\end{array}$ & Buses & $\begin{array}{c}\text { Class } \\
3-6 \\
\text { trucks }\end{array}$ & $\begin{array}{c}\text { Class } \\
7-8 \\
\text { trucks } \\
\end{array}$ & $\begin{array}{l}\text { Heavy } \\
\text { trucks } \\
\text { subtotal }\end{array}$ & & $\begin{array}{c}\text { Highway } \\
\text { subtotal }\end{array}$ & $\begin{array}{c}\text { Total } \\
\text { transportation }\end{array}$ \\
\hline 1970 & 10,513 & 1,906 & 12,419 & 9 & 153 & 403 & 1,452 & 1,855 & & 14,437 & 18,698 \\
\hline 1975 & 11,514 & 2,953 & 14,467 & 17 & 147 & 520 & 1,873 & 2,393 & & 17,025 & 21,153 \\
\hline 1976 & 12,164 & 3,223 & 15,388 & 19 & 159 & 549 & 1,977 & 2,526 & & 18,092 & 22,438 \\
\hline 1977 & 12,287 & 3,464 & 15,751 & 20 & 164 & 608 & 2,192 & 2,801 & & 18,735 & 23,259 \\
\hline 1978 & 12,539 & 3,739 & 16,279 & 22 & 168 & 817 & 2,304 & 3,122 & & 19,590 & 24,426 \\
\hline 1979 & 11,912 & 3,783 & 15,695 & 27 & 172 & 993 & 2,244 & 3,237 & & 19,130 & 23,877 \\
\hline 1980 & 10,886 & 3,683 & 14,569 & 32 & 171 & 1,137 & 2,093 & 3,231 & & 18,002 & 22,990 \\
\hline 1981 & 10,753 & 3,667 & 14,420 & 33 & 173 & 1,306 & 1,978 & 3,284 & & 17,911 & 22,761 \\
\hline 1982 & 10,742 & 3,513 & 14,255 & 31 & 180 & 1,452 & 1,819 & 3,271 & & 17,737 & 22,185 \\
\hline 1983 & 10,917 & 3,704 & 14,621 & 27 & 181 & 1,376 & 1,966 & 3,342 & & 18,170 & 22,372 \\
\hline 1984 & 10,966 & 3,963 & 14,929 & 27 & 175 & 1,314 & 2,146 & 3,460 & & 18,591 & 23,086 \\
\hline 1985 & 11,095 & 4,233 & 15,328 & 28 & 182 & 1,206 & 2,261 & 3,467 & & 19,005 & 23,399 \\
\hline 1986 & 11,351 & 4,501 & 15,851 & 29 & 192 & 1,124 & 2,428 & 3,552 & & 19,624 & 24,673 \\
\hline 1987 & 11,371 & 4,735 & 16,106 & 29 & 196 & 1,046 & 2,623 & 3,669 & & 20,001 & 25,256 \\
\hline 1988 & 11,376 & 5,057 & 16,433 & 31 & 203 & 1,048 & 2,689 & 3,737 & & 20,404 & 25,929 \\
\hline 1989 & 11,464 & 5,153 & 16,617 & 32 & 202 & 1,058 & 2,775 & 3,833 & & 20,685 & 26,355 \\
\hline 1990 & 10,790 & 5,521 & 16,311 & 30 & 199 & 1,085 & 2,909 & 3,993 & & 20,533 & 26,221 \\
\hline 1991 & 9,976 & 5,924 & 15,900 & 28 & 212 & 1,089 & 2,985 & 4,074 & & 20,215 & 25,736 \\
\hline 1992 & 10,149 & 6,351 & 16,500 & 30 & 220 & 1,091 & 3,061 & 4,152 & & 20,901 & 26,538 \\
\hline 1993 & 10,399 & 6,649 & 17,049 & 31 & 218 & 1,101 & 3,181 & 4,282 & & 21,579 & 27,140 \\
\hline 1994 & 10,528 & 6,847 & 17,375 & 32 & 218 & 1,137 & 3,384 & 4,520 & & 22,145 & 27,879 \\
\hline 1995 & 10,557 & 7,074 & 17,631 & 31 & 219 & 1,157 & 3,552 & 4,709 & & 22,590 & 28,528 \\
\hline 1996 & 10,735 & 7,348 & 18,083 & 30 & 222 & 1,161 & 3,676 & 4,838 & & 23,173 & 29,153 \\
\hline 1997 & 10,837 & 7,666 & 18,503 & 31 & 229 & 1,145 & 3,740 & 4,885 & & 23,648 & 29,602 \\
\hline 1998 & 11,116 & 7,830 & 18,946 & 32 & 234 & 1,172 & 3,871 & 5,043 & & 24,254 & 30,013 \\
\hline 1999 & 11,362 & 8,199 & 19,561 & 33 & 241 & 1,277 & 4,267 & 5,544 & & 25,379 & 31,572 \\
\hline 2000 & 11,326 & 8,209 & 19,536 & 32 & 248 & 1,314 & 4,445 & 5,760 & & 25,576 & 31,943 \\
\hline 2001 & 11,403 & 8,297 & 19,700 & 30 & 233 & 1,301 & 4,451 & 5,751 & & 25,714 & 31,581 \\
\hline 2002 & 11,699 & 8,557 & 20,256 & 30 & 228 & 1,348 & 4,668 & 6,016 & & 26,530 & 32,319 \\
\hline 2003 & 11,561 & 9,414 & 20,975 & 30 & 226 & 1,311 & 4,538 & 5,849 & & 27,079 & 32,619 \\
\hline 2004 & 11,685 & 9,825 & 21,510 & 31 & 230 & 1,214 & 4,205 & 5,419 & & 27,191 & 33,216 \\
\hline 2005 & 11,997 & 9,120 & 21,117 & 29 & 233 & 1,362 & 4,718 & 6,080 & & 27,460 & 33,684 \\
\hline 2006 & 11,626 & 9,403 & 21,029 & 34 & 237 & 1,391 & 4,815 & 6,206 & $\mathrm{~b}$ & 27,506 & 33,808 \\
\hline 2007 & 11,527 & 9,581 & 21,108 & 74 & 233 & 1,730 & 5,990 & 7,719 & & 29,133 & 35,584 \\
\hline 2008 & 11,079 & 9,480 & 20,559 & 76 & 239 & 1,748 & 6,051 & 7,799 & & 28,673 & 34,876 \\
\hline 2009 & 10,317 & 9,576 & 19,893 & 75 & 239 & 1,623 & 5,620 & 7,243 & & 27,449 & 33,119 \\
\hline 2010 & 9,643 & 10,019 & 19,662 & 66 & 228 & 1,649 & 5,711 & 7,361 & & 27,316 & 33,221 \\
\hline 2011 & 9,241 & 10,189 & 19,430 & 66 & 233 & 1,553 & 5,378 & 6,931 & & 26,660 & 32,512 \\
\hline 2012 & 8,971 & 10,283 & 19,254 & 76 & 240 & 1,551 & 5,372 & 6,924 & & 26,494 & 32,040 \\
\hline 2013 & 8,879 & 10,153 & 19,032 & 72 & 245 & 1,586 & 5,492 & 7,078 & & 26,428 & 31,922 \\
\hline 2014 & 8,751 & 10,685 & 19,436 & 71 & 246 & 1,612 & 5,583 & 7,195 & & 26,949 & 32,166 \\
\hline 2015 & 8,556 & 10,983 & 19,539 & 69 & 251 & 1,602 & 5,548 & 7,150 & & 27,009 & 32,383 \\
\hline 2016 & 8,409 & 11,318 & 19,726 & 72 & 256 & 1,645 & 5,695 & 7,339 & & 27,394 & 32,919 \\
\hline 2017 & 8,107 & 11,429 & 19,537 & 71 & 264 & 1,684 & 5,830 & 7,514 & & 27,386 & 33,049 \\
\hline & & \multicolumn{10}{|c|}{ Average annual percentage change } \\
\hline $1970-2017$ & $-0.6 \%$ & $3.9 \%$ & $1.0 \%$ & $4.4 \%$ & $1.2 \%$ & $3.1 \%$ & $3.0 \%$ & $3.0 \%$ & & $1.4 \%$ & $1.2 \%$ \\
\hline $2007-2017$ & $-3.5 \%$ & $1.8 \%$ & $-0.8 \%$ & $-0.4 \%$ & $1.3 \%$ & $-0.3 \%$ & $-0.3 \%$ & $-0.3 \%$ & & $-0.6 \%$ & $-0.7 \%$ \\
\hline
\end{tabular}

Source: Vehicle energy use from the Data Book plus upstream energy use for all fuels.

${ }^{a}$ Total transportation figures do not include military and off-highway energy use and may not include all possible uses of fuel for transportation (e.g., snowmobiles).

${ }^{\mathrm{b}}$ Due to changes in the FHWA fuel use methodology, motorcycle, bus, and heavy truck data are not comparable with data before the year 2007. 
Table 8. Nonhighway Transportation Energy Consumption Including Upstream Energy by Mode, 1970-2017

(trillion Btu)

\begin{tabular}{|c|c|c|c|c|c|c|}
\hline Year & Air & Water & Pipeline & Rail & $\begin{array}{c}\text { Nonhighway } \\
\text { subtotal }\end{array}$ & $\begin{array}{c}\text { Total } \\
\text { transportation }^{\mathrm{a}}\end{array}$ \\
\hline 1970 & 1,486 & 974 & 1,145 & 655 & 4,261 & 18,698 \\
\hline 1975 & 1,423 & 1,081 & 964 & 660 & 4,127 & 21,153 \\
\hline 1976 & 1,482 & 1,260 & 918 & 686 & 4,346 & 22,438 \\
\hline 1977 & 1,557 & 1,368 & 900 & 698 & 4,524 & 23,259 \\
\hline 1978 & 1,642 & 1,603 & 895 & 695 & 4,835 & 24,426 \\
\hline 1979 & 1,718 & 1,327 & 985 & 717 & 4,746 & 23,877 \\
\hline 1980 & 1,655 & 1,609 & 1,030 & 694 & 4,988 & 22,990 \\
\hline 1981 & 1,677 & 1,470 & 1,041 & 662 & 4,850 & 22,761 \\
\hline 1982 & 1,666 & 1,233 & 982 & 567 & 4,449 & 22,185 \\
\hline 1983 & 1,660 & 1,132 & 847 & 563 & 4,202 & 22,372 \\
\hline 1984 & 1,856 & 1,120 & 895 & 625 & 4,495 & 23,086 \\
\hline 1985 & 1,934 & 1,013 & 863 & 584 & 4,394 & 23,399 \\
\hline 1986 & 2,101 & 1,540 & 839 & 569 & 5,049 & 24,673 \\
\hline 1987 & 2,188 & 1,604 & 882 & 581 & 5,255 & 25,256 \\
\hline 1988 & 2,279 & 1,650 & 1,001 & 595 & 5,525 & 25,929 \\
\hline 1989 & 2,282 & 1,763 & 1,024 & 600 & 5,670 & 26,355 \\
\hline 1990 & 2,357 & 1,678 & 1,062 & 590 & 5,688 & 26,221 \\
\hline 1991 & 2,207 & 1,770 & 988 & 556 & 5,521 & 25,736 \\
\hline 1992 & 2,240 & 1,858 & 968 & 570 & 5,636 & 26,538 \\
\hline 1993 & 2,286 & 1,672 & 1,014 & 588 & 5,561 & 27,140 \\
\hline 1994 & 2,389 & 1,624 & 1,093 & 628 & 5,734 & 27,879 \\
\hline 1995 & 2,465 & 1,709 & 1,112 & 652 & 5,938 & 28,528 \\
\hline 1996 & 2,540 & 1,645 & 1,127 & 667 & 5,980 & 29,153 \\
\hline 1997 & 2,648 & 1,462 & 1,175 & 668 & 5,953 & 29,602 \\
\hline 1998 & 2,620 & 1,441 & 1,026 & 672 & 5,759 & 30,013 \\
\hline 1999 & 2,859 & 1,599 & 1,039 & 697 & 6,193 & 31,572 \\
\hline 2000 & 2,940 & 1,696 & 1,034 & 696 & 6,367 & 31,943 \\
\hline 2001 & 2,760 & 1,390 & 1,016 & 700 & 5,866 & 31,581 \\
\hline 2002 & 2,566 & 1,457 & 1,065 & 701 & 5,790 & 32,319 \\
\hline 2003 & 2,601 & 1,256 & 967 & 716 & 5,540 & 32,619 \\
\hline 2004 & 2,827 & 1,513 & 932 & 754 & 6,025 & 33,216 \\
\hline 2005 & 2,918 & 1,591 & 954 & 761 & 6,224 & 33,684 \\
\hline 2006 & 2,892 & 1,681 & 953 & 776 & 6,301 & 33,808 \\
\hline 2007 & 2,889 & 1,803 & 999 & 759 & 6,451 & 35,584 \\
\hline 2008 & 2,759 & 1,681 & 1,033 & 731 & 6,203 & 34,876 \\
\hline 2009 & 2,449 & 1,544 & 1,058 & 618 & 5,669 & 33,119 \\
\hline 2010 & 2,473 & 1,702 & 1,063 & 667 & 5,905 & 33,221 \\
\hline 2011 & 2,483 & 1,590 & 1,080 & 699 & 5,852 & 32,512 \\
\hline 2012 & 2,391 & 1,341 & 1,129 & 684 & 5,546 & 32,040 \\
\hline 2013 & 2,344 & 1,190 & 1,261 & 699 & 5,494 & 31,922 \\
\hline 2014 & 2,371 & 1,029 & 1,090 & 728 & 5,218 & 32,166 \\
\hline 2015 & 2,437 & 1,181 & 1,057 & 698 & 5,373 & 32,383 \\
\hline 2016 & 2,507 & 1,305 & 1,065 & 647 & 5,524 & 32,919 \\
\hline 2017 & 2,568 & 1,320 & 1,109 & 666 & 5,664 & 33,049 \\
\hline \multicolumn{7}{|c|}{ Average annual percentage change } \\
\hline 1970-2017 & $1.2 \%$ & $0.6 \%$ & $-0.1 \%$ & $0.0 \%$ & $0.6 \%$ & $1.2 \%$ \\
\hline $2007-2017$ & $-1.2 \%$ & $-3.1 \%$ & $1.0 \%$ & $-1.3 \%$ & $-1.3 \%$ & $-0.7 \%$ \\
\hline
\end{tabular}

Source: Vehicle energy use from the Data Book plus upstream energy use for all fuels.

a Total transportation figures do not include military and off-highway energy use and may not include all possible uses of fuel for transportation (e.g., snowmobiles). 


\section{COMPARISON OF TRANSPORATION ENERGY CONSUMPTION WITH AND WITHOUT UPSTREAM ENERGY}

By including upstream energy into transportation energy consumption, total energy use changed from 26.6 quads to 33.0 quads. A graphical comparison of transportation energy consumption with and without upstream energy by mode and fuel type is shown in Figures 1 and 2. Neither the modal shares nor the fuel shares were greatly affected by including upstream energy, except for rail and pipeline. These two modes have the highest electricity consumption and that is where the largest differences lie.

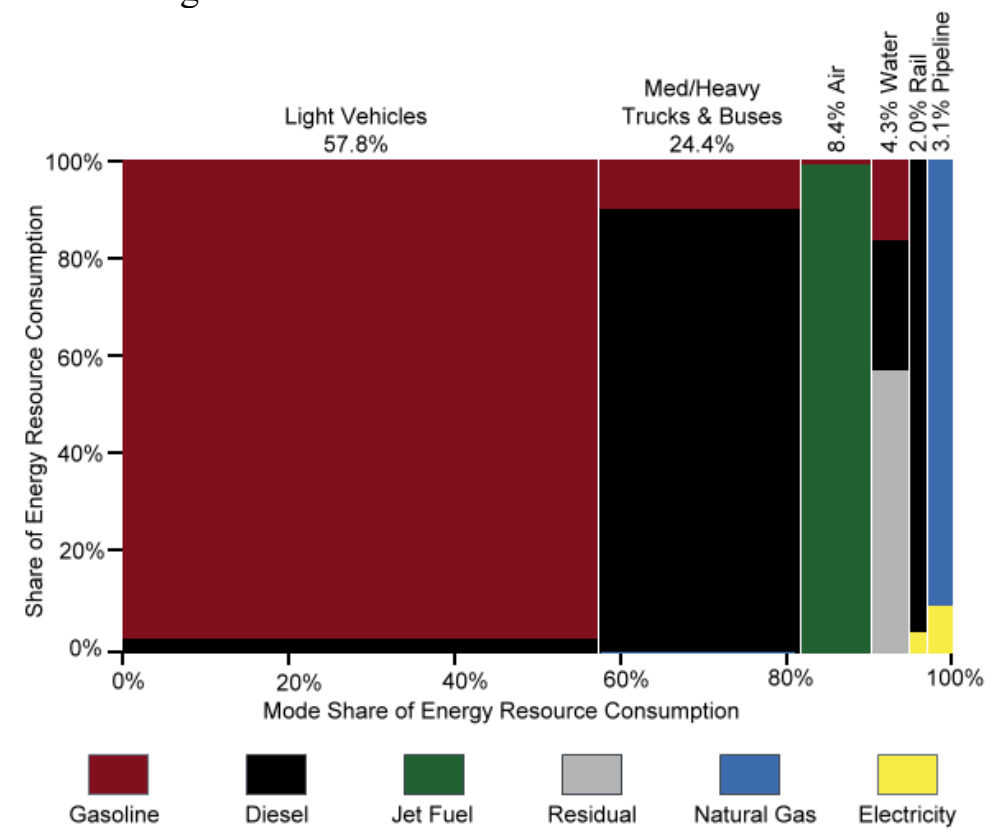

Figure 1. Transportation energy consumption without upstream energy included.

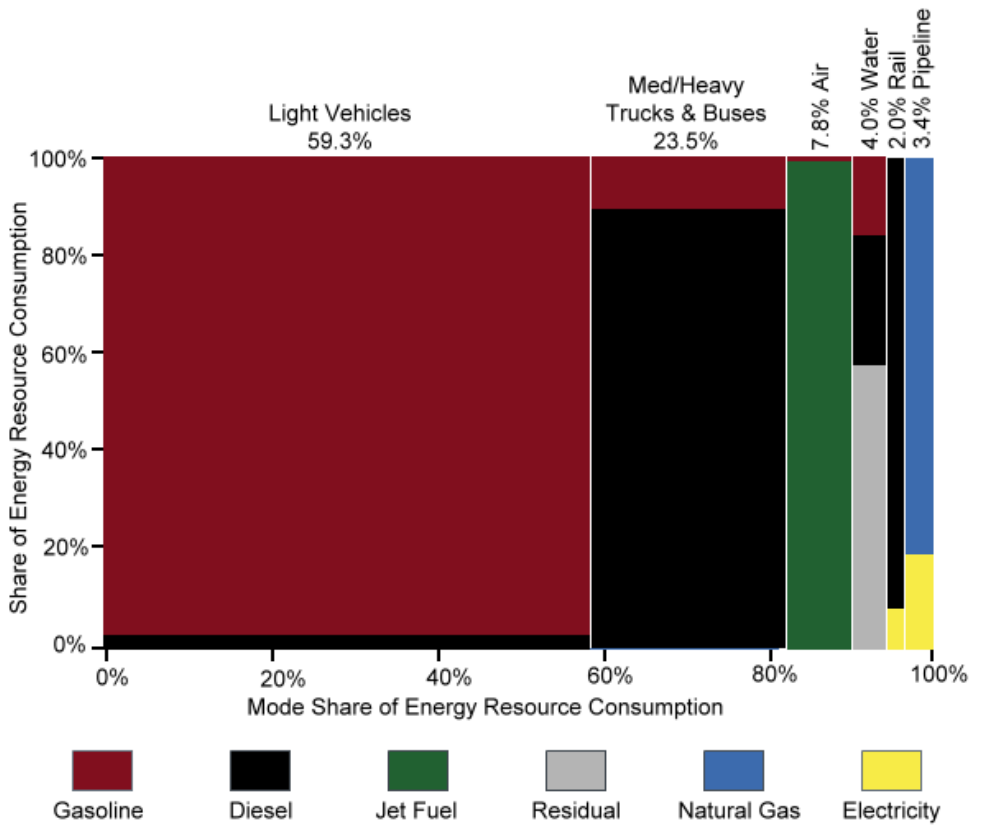

Figure 2. Transportation energy consumption with upstream energy included. 
A full comparison of the data with and without upstream energy by mode is shown on Table 9, along with the modal shares for each. The modal shares are quite similar between the two data series. The differences in the modal shares are less than $1 \%$ for each mode with one exception. The light vehicle subcategory increased by $1.5 \%$ when including upstream energy.

Table 9. Transportation Energy Use With and Without Upstream Energy by Mode, 2017

\begin{tabular}{|c|c|c|c|c|}
\hline & \multicolumn{2}{|c|}{ Trillion Btu } & \multicolumn{2}{|c|}{ Percentage of total based on Btus } \\
\hline & Vehicle Only & With Upstream & Vehicle Only & With Upstream \\
\hline HIGHWAY & $21,868.7$ & $27,385.9$ & $82.2 \%$ & $82.9 \%$ \\
\hline Light vehicles & $15,359.8$ & $19,608.2$ & $\mathbf{5 7 . 8 \%}$ & $59.3 \%$ \\
\hline Cars & $6,339.3$ & $8,107.4$ & $23.8 \%$ & $24.5 \%$ \\
\hline Light trucks ${ }^{\mathrm{b}}$ & $8,963.2$ & $11,429.5$ & $33.7 \%$ & $34.6 \%$ \\
\hline Motorcycles & 57.3 & 71.3 & $0.2 \%$ & $0.2 \%$ \\
\hline Buses & 220.3 & 263.5 & $0.8 \%$ & $0.8 \%$ \\
\hline Transit & 91.6 & 109.9 & $0.3 \%$ & $0.3 \%$ \\
\hline Intercity & 37.3 & 44.4 & $0.1 \%$ & $0.1 \%$ \\
\hline School & 91.4 & 109.2 & $0.3 \%$ & $0.3 \%$ \\
\hline Medium/heavy trucks & $6,288.6$ & $7,514.2$ & $23.6 \%$ & $22.7 \%$ \\
\hline Class 3-6 trucks & $1,391.2$ & $1,683.8$ & $5.2 \%$ & $5.1 \%$ \\
\hline Class 7-8 trucks & $4,897.4$ & $5,830.4$ & $18.4 \%$ & $17.6 \%$ \\
\hline NONHIGHWAY & $4,723.2$ & $5,663.5$ & $17.8 \%$ & $17.1 \%$ \\
\hline Air & $2,230.8$ & $2,568.2$ & $8.4 \%$ & $7.8 \%$ \\
\hline General aviation & 231.7 & 269.1 & $0.9 \%$ & $0.8 \%$ \\
\hline Domestic air carriers & $1,564.3$ & $1,799.0$ & $5.9 \%$ & $5.4 \%$ \\
\hline International air & 434.8 & 500.1 & $1.6 \%$ & $1.5 \%$ \\
\hline Water & $1,130.4$ & $1,320.2$ & $4.3 \%$ & $4.0 \%$ \\
\hline Freight & 920.2 & $1,061.5$ & $3.5 \%$ & $3.2 \%$ \\
\hline Recreational & 210.3 & 258.7 & $0.8 \%$ & $0.8 \%$ \\
\hline Pipeline & 825.0 & $1,109.1$ & $3.1 \%$ & $3.4 \%$ \\
\hline Rail & 536.9 & 666.0 & $2.0 \%$ & $2.0 \%$ \\
\hline Freight (Class I) & 490.5 & 583.7 & $1.8 \%$ & $1.8 \%$ \\
\hline Passenger & 46.4 & 82.3 & $0.2 \%$ & $0.2 \%$ \\
\hline Transit & 15.9 & 34.3 & $0.1 \%$ & $0.1 \%$ \\
\hline Commuter & 20.5 & 33.6 & $0.1 \%$ & $0.1 \%$ \\
\hline Intercity & 10.0 & 14.4 & $0.0 \%$ & $0.0 \%$ \\
\hline HWY \& NONHWY TOTAL & $26,591.9$ & $33,049.4$ & $100.0 \%$ & $100.0 \%$ \\
\hline
\end{tabular}

Source: Tables 2 and 6.

a Civilian consumption only. Totals may not include all possible uses of fuels for transportation (e.g., snowmobiles).

b Two-axle, four-tire trucks. 
Figure 3 shows the percent change from the vehicle-only energy use to the energy use including upstream energy by mode, while Figure 4 shows the percent change by fuel type. Again, the modes using electricity, pipeline and passenger rail, show the largest changes by mode, and electricity shows the largest changes of the fuel types.

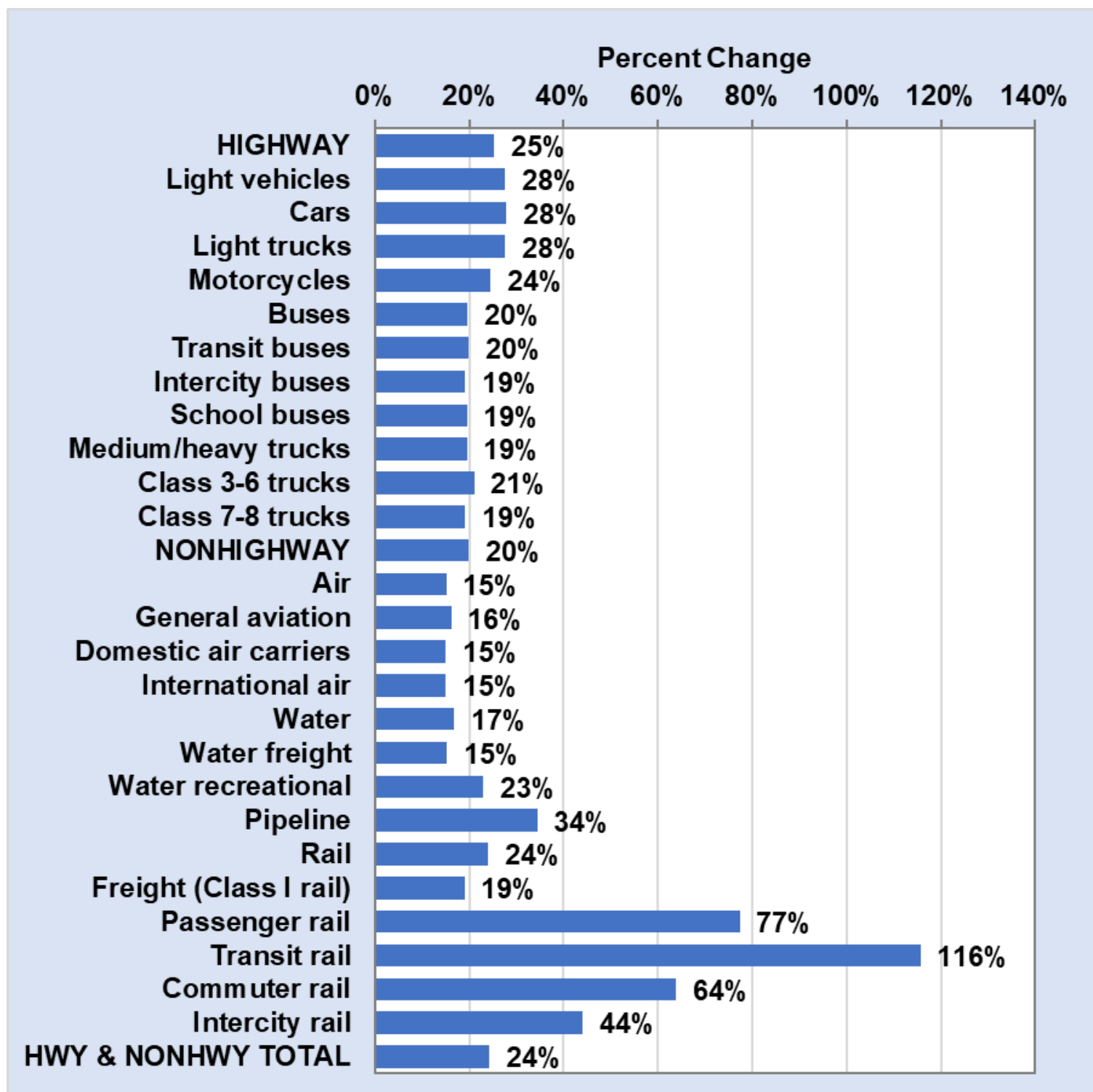

Figure 3. Percent change of vehicle energy use to energy use including upstream energy by mode, 2017. 


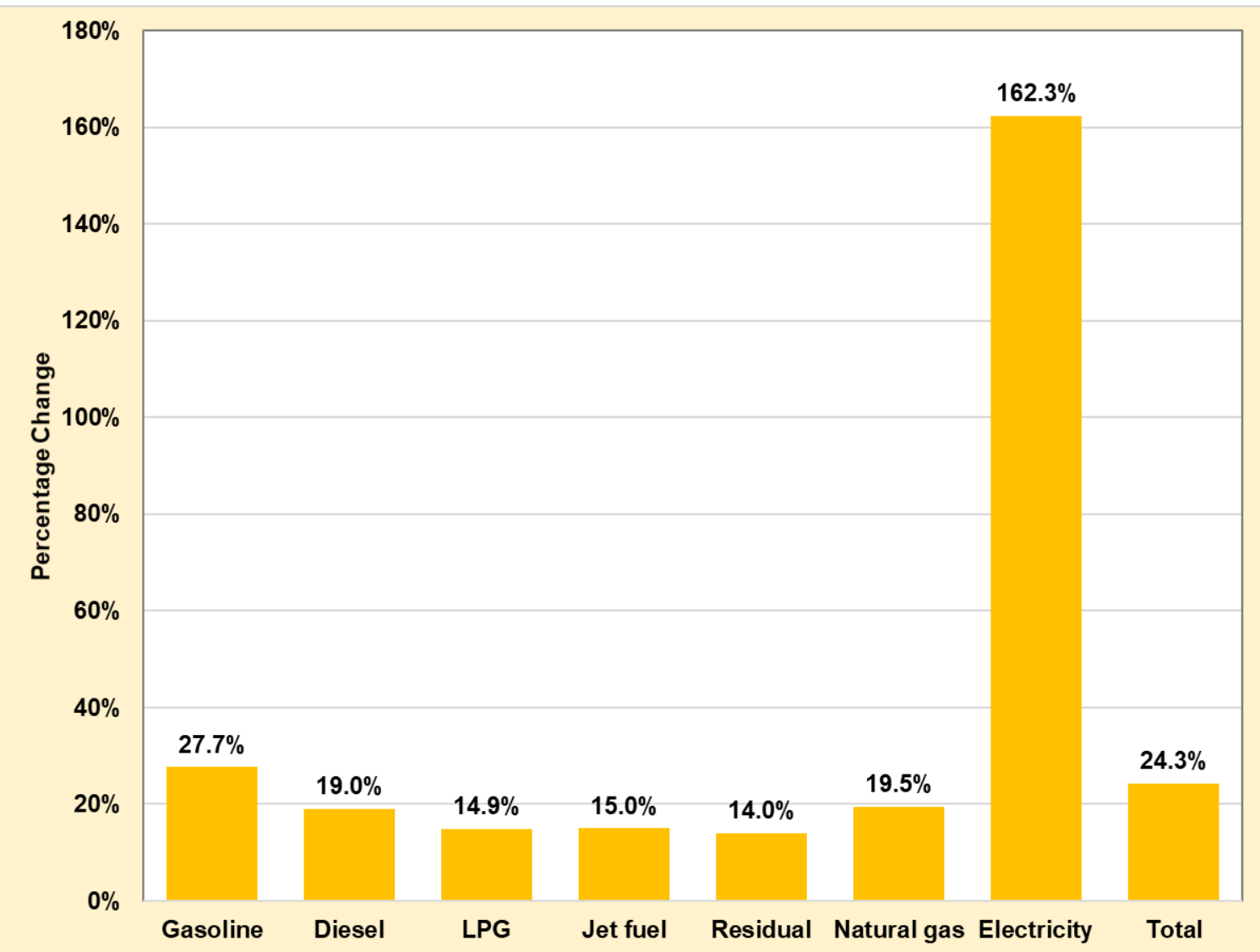

Figure 4. Percent change of vehicle energy use to energy use including upstream energy by fuel type, 2017. 
In terms of historical changes, the two series mirror each other (Figure 5). This was expected because, except for electricity, the factors used to include upstream energy were the same for every year (Table 1 for electricity and Table 5 for all other fuels). The GREET calculator does not include unique estimates of upstream energy use for historical years, thus the 2019 factors were used historically.

The gap between the two data series is widening. In 1970 the two lines in Figure 5 differ by $3,505 \mathrm{Btu}$ and in 2017, by 6,457 Btu. The greater use of fuels that have higher upstream energy use, like electricity and gasoline, is the reason for the gap increase. The gap would be even wider if not for the increase in efficiency for electricity net generation (Table 1).

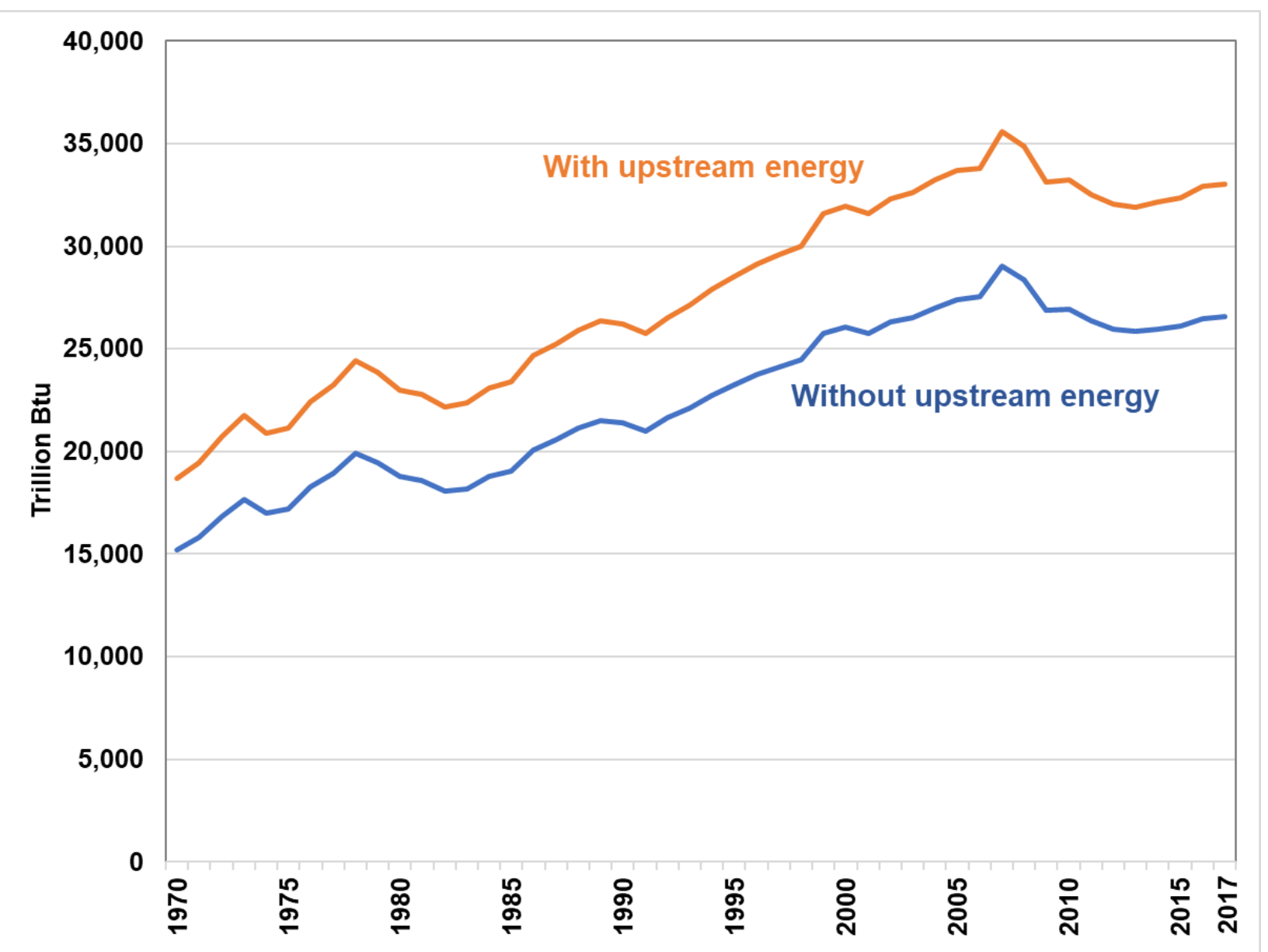

Figure 5. Historical transportation energy consumption with and without upstream energy, 19702017. 


\section{CONCLUSIONS}

A comparison of transportation energy use with and without upstream energy by mode and fuel type was performed for the Data Book's big energy table. The data to include upstream energy for electricity came from the Energy Information Administration's Monthly Energy Review and for other fuels came from ANL's GREET model WTW 2019 Calculator. Including upstream energy added 24\% (6.4 quads) to the vehicle fuel consumption that is published on Table 2.7 of the Data Book. For most of the transportation modes, the share of consumption by mode and fuel type looked the same whether including upstream energy or not. However, the modes with the largest use of electricity, rail and pipeline, showed differences in fuel mix due to the high upstream energy use for that fuel. Electricity use increased by $162 \%$ when upstream energy was added. Gasoline, the second highest in percent change, increased by $28 \%$ with the addition of upstream energy. When including upstream energy, total transit rail energy use increased by $116 \%$, commuter rail by $64 \%$, and intercity rail by $44 \%$. Pipeline was the only other mode with over a 30\% increase. Historically, the trend with upstream energy included and without show similar trends with a gradual widening of the gap between the two data series. The greater use of fuels that have higher upstream energy use, like electricity and gasoline, is the reason for the gap increase. As the highway sector transitions towards heavier use of electricity, the differences between including and excluding upstream energy will become more pronounced. 